\title{
Dielectric Properties of Strongly Polar Nematogens
}

\author{
Joanna Czub ${ }^{\mathrm{a}}$, Roman Dạbrowski ${ }^{\mathrm{b}}$, Stanisław Urban ${ }^{\mathrm{c}}$, and Dorota Ziobro ${ }^{\mathrm{b}}$ \\ ${ }^{a}$ Faculty of Metals Engineeing and Industrial Computer Science, AGH University of Technology, \\ Al. Mickiewicza 30, 30-059 Kraków, Poland \\ ${ }^{\mathrm{b}}$ Institute of Chemistry, Military University of Technology, Kaliskiego 2, 00-908 Warsaw, Poland

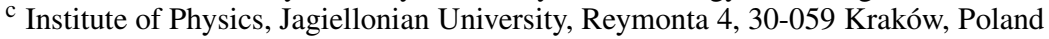 \\ Reprint requests to Dr. J. C.; Fax: 0048-12-6173190; E-mail: joanna.czub@agh.edu.pl
}

Z. Naturforsch. 65a, 221 - 230 (2010); received January 16, 2009 / revised June 3, 2009

\begin{abstract}
Results of the static and dynamic dielectric investigations of four liquid crystalline compounds consisting of two or three phenyl rings with $n$-alkoxy and cyano terminals, carboxylic bridging groups, and fluoro lateral substituents in pure state and in the solution of low polar liquid crystalline medium, are presented. These compounds form the nematic phase being a monotropic one in the case of two ring compounds. The dielectric parameters are analyzed in relation to the dipolar structures of the molecules. The dielectric properties of pure substances are strongly influenced by conductivity and dimerization effects, while extracted from the data for solutions are consisted well with the structures. In the nematic phase the compounds exhibit a crossover of the principal permittivity components among the kilo and megahertz frequency range.
\end{abstract}

Key words: Liquid Crystals; Nematic Phase; Dielectric Properties; Dipolar Structure.

\section{Introduction}

Dielectric relaxation studies of liquid crystals (LC) yield important information on molecular reorientations and their interactions in mesophases [1-4]. Knowledge of the dielectric properties of LCs is also important for the technical reasons due to their connection with electrooptical display devices as they strongly influence threshold voltage and steepness of electrooptical curves [5-7].

The aim of the present work was to analyze the influence of rigid core length, terminal chain length, and dipole moment value on the dielectric properties of four substances having similar chemical structures (Table 1). All substances are nematogens, although two-ring compounds exhibit a monotropic nematic $(\mathrm{N})$ phase only. They have several dipolar groups $\left(-\mathrm{OC}_{\mathrm{n}} \mathrm{H}_{2 \mathrm{n}+1}\right.$ and $-\mathrm{CN}$ terminals, $\mathrm{F}$ atoms at the lateral positions, $-\mathrm{COO}$ bridging groups) which create strong longitudinal and transverse dipole moment components. This should be reflected in the dielectric properties of the isotropic and nematic phases observed at low frequencies (static case) as well as at high frequencies (dynamical case). Such a compound structure is especially interesting for some applications [7]. They belong to the class of materials termed as dual frequency materials exhibiting a crossover in electric permittivity constants at a certain frequency what leads to a change of sign of the dielectric anisotropy [6,7].

It was found that such compounds, especially the three-ring ones, exhibited a large conductivity effect at low frequencies in spite of purification the chromatography column filled with silica-gel and several recrystallization procedures repeated. It is known from calculations carried out by Demus et al. [8] that for a LC medium with dielectric anisotropy $\Delta \varepsilon>10$ the resistivity starts to decrease probably because of dissociation of polar bonds and inclusion of ions. Therefore, for those substances the static permittivity components were determined also from the studies of their $10 \% \mathrm{wt}$. solution in the Base 903 ester mixture showing a small negative dielectric anisotropy (Table 1). Similar studies were reported by Heppke et al. [9].

\section{Experimental}

All substances were synthesized in the Institute of Chemistry, Military University of Technology, Warsaw, according to the route described in $[10,11]$.

The dielectric relaxation spectra, $\varepsilon^{*}(f)=\varepsilon^{\prime}(f)-$ i $\varepsilon^{\prime \prime}(f)$, were recorded with the aid of an impedance analyzer HP 4192A in the frequency range of $1 \mathrm{kHz}-$ $30 \mathrm{MHz}$. The parallel plate capacitor was used $\left(C_{\mathrm{o}} \sim\right.$ $50 \mathrm{pF}$ ). The thickness of the samples was $0.7 \mathrm{~mm}$. 


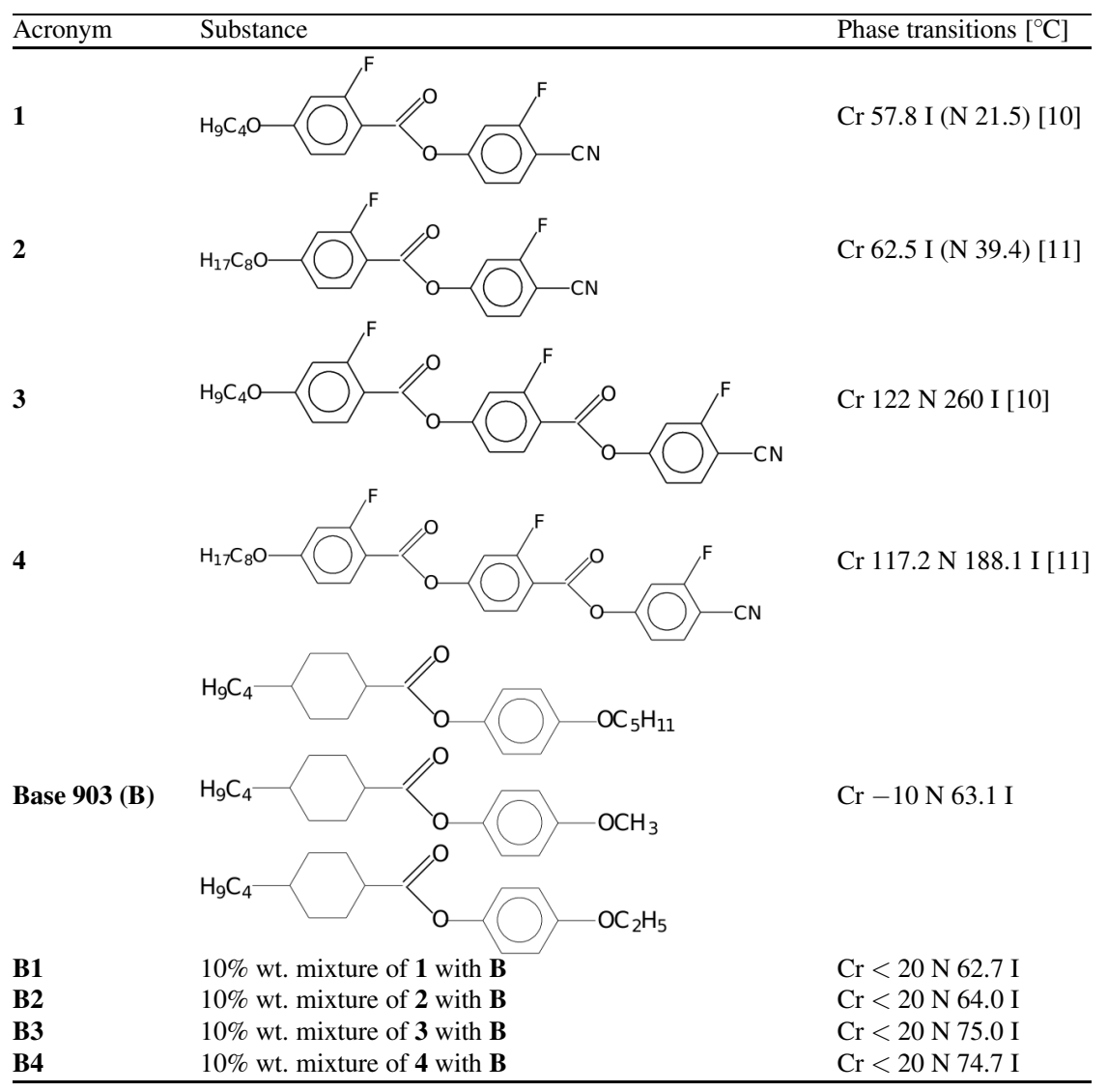

The samples in the $\mathrm{N}$ phase were oriented by a magnetic field of $0.8 \mathrm{~T}$. Two experimental geometries were applied: $\mathbf{E} \| \mathbf{B} \equiv \mathbf{n}$ and $\mathbf{E} \perp \mathbf{B} \equiv \mathbf{n}(\mathbf{n}-$ director, $\mathbf{E}-$ measuring electric field), enabling the measurement of the $\varepsilon_{\|}$and $\varepsilon_{\perp}$ permittivity tensor components, respectively. The temperature was stabilized within $\pm 0.1 \mathrm{~K}$ in the range of $20{ }^{\circ} \mathrm{C}-90{ }^{\circ} \mathrm{C}$ and $\pm 0.2 \mathrm{~K}$ in the range of $90{ }^{\circ} \mathrm{C}-160{ }^{\circ} \mathrm{C}$. In the isotropic phase a time domain spectrometer (TDS) was employed that covers the frequency range of $10 \mathrm{MHz}-3 \mathrm{GHz}$ [12]. All measurements were carried out with decreasing temperature which caused supercooling of the $\mathrm{N}$ phase. In substances $\mathbf{1}$ and $\mathbf{2}$ a monotropic $\mathrm{N}$ phase appeared.

\section{Results}

\subsection{Two-Ring Compounds}

Figure 1 presents a comparisons of the tensor permittivity components, $\varepsilon_{\|}, \varepsilon_{\perp}$, and $\bar{\varepsilon}=\left(\varepsilon_{\|}+2 \varepsilon_{\perp}\right) / 3$, obtained for two-ring compounds. The monotropic $\mathrm{N}$ phase could be supercooled by c. six degrees only
Table 1. Chemical formulae and phase transition temperatures of the substances studied.

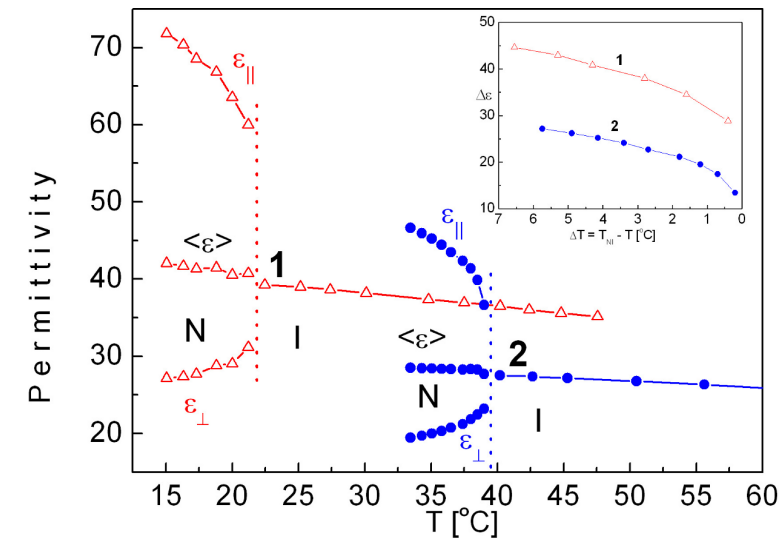

Fig. 1. Static permittivity components for compounds $\mathbf{1}$ and 2 . The inset shows the dielectric anisotropy $\Delta \varepsilon=\varepsilon_{\|}-\varepsilon_{\perp}$ versus the shifted temperature $\Delta T=T_{N I}-T$.

before a rapid crystallization. The relaxation spectra collected in the isotropic and nematic phases of both substances are shown in Figures 2 and 3 in form of the Cole-Cole plots [13]. The superposition of the Debye- 

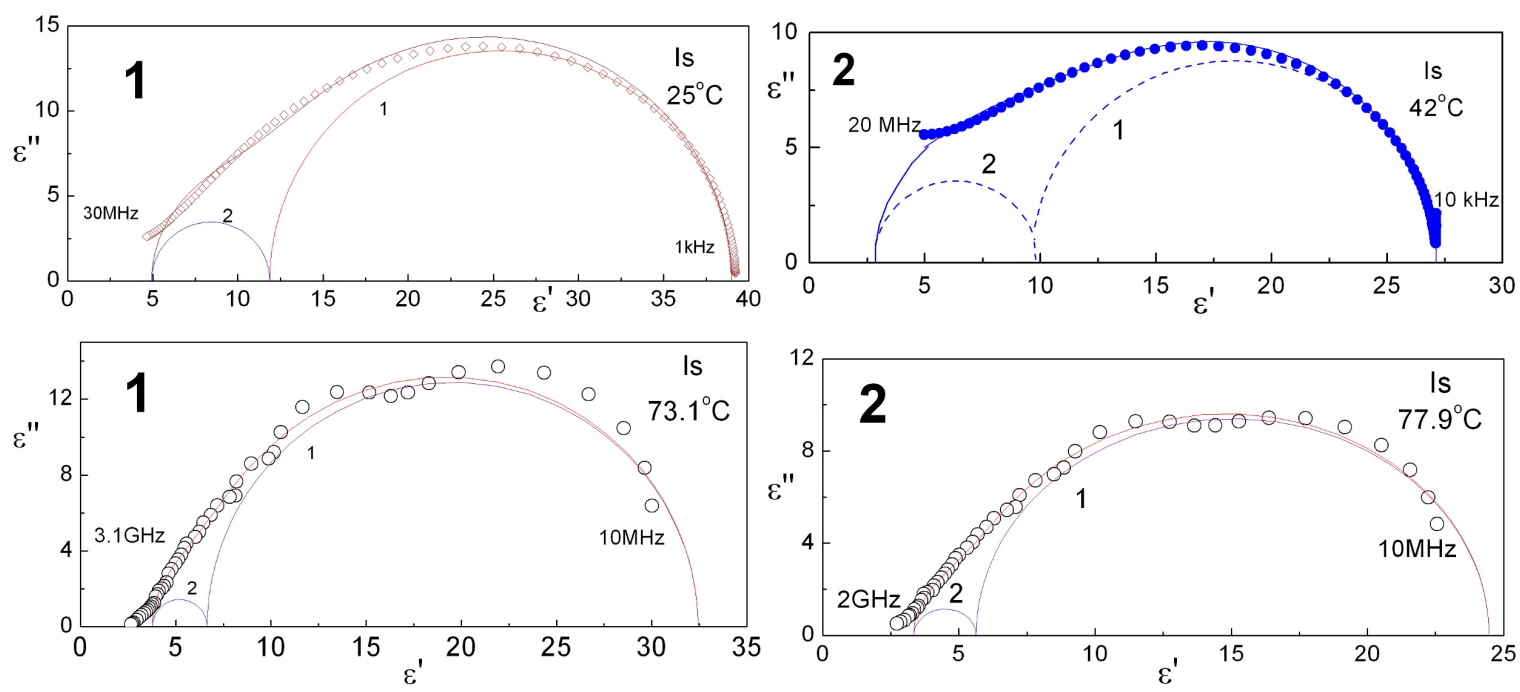

Fig. 2. Cole-Cole plots for the isotropic phase of two-ring substances $\mathbf{1}$ and $\mathbf{2}$. The spectra in the upper row were collected at temperatures close to the clearing point with the aid of an impedance analyzer, in the bottom row by the TDS method in the high temperature region of the isotropic phase.
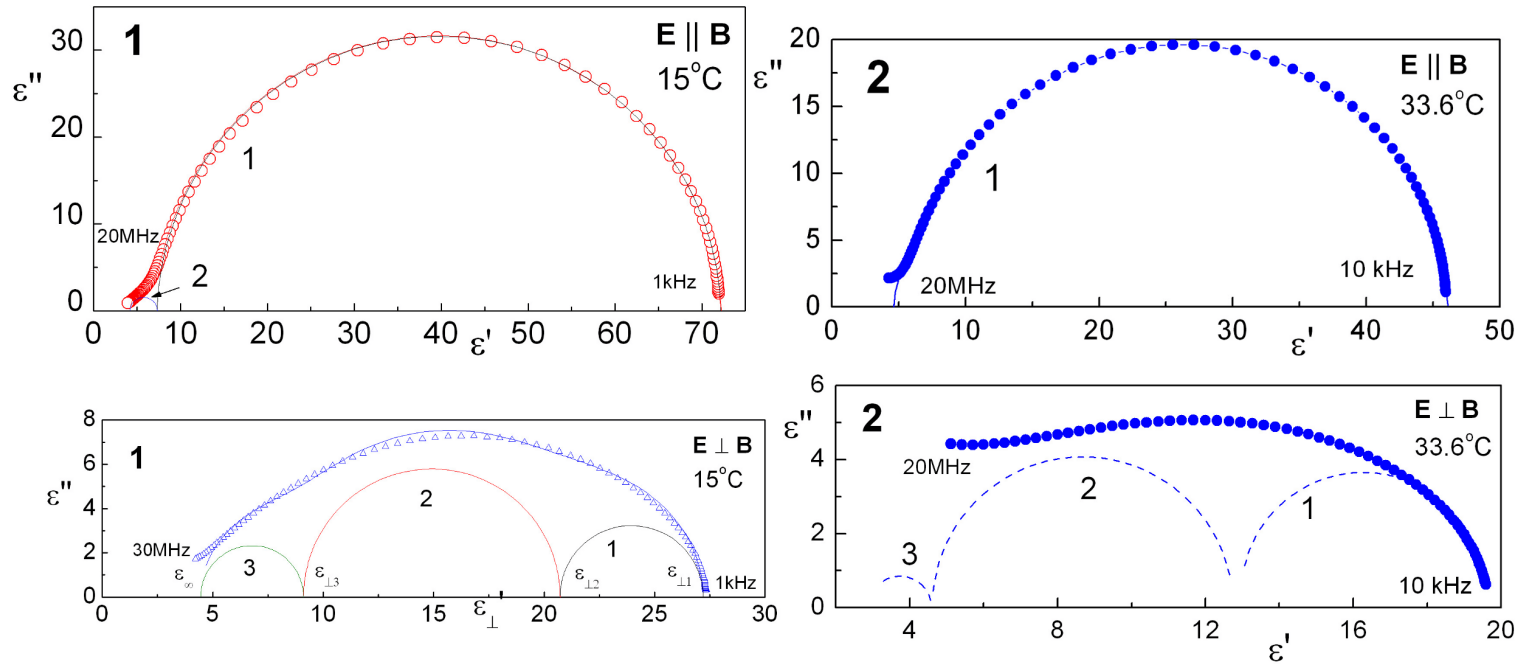

Fig. 3. Cole-Cole plots for two-ring substances in the nematic phase orientated parallel (upper row) and perpendicular (lower row) to the measuring field. The spectra were collected with the aid of an impedance analyzer.

type processes well fits the experimental points,

$$
\varepsilon^{*}(\omega)=\varepsilon^{\prime}(\omega)-\mathrm{i} \varepsilon^{\prime \prime}(\omega)=\sum_{k} \frac{\varepsilon_{\mathrm{s}}-\varepsilon_{\infty k}}{1+\mathrm{i} \omega \tau_{k}}-\mathrm{i} \frac{\sigma(\omega)}{\varepsilon_{0} \omega},
$$

where $\varepsilon_{\mathrm{s}}$ and $\varepsilon_{\infty}$ stand for the static and high frequency permittivities, respectively, $\tau$ is the relaxation time, $\sigma(\omega)$ the ionic conductivity, $\omega=2 \pi f$ is a circular frequency, $k$ numbers the relaxation processes, $\varepsilon_{\mathrm{s}}-\varepsilon_{\infty}$ is called the strength of the relaxation process or the dielectric increment.

\subsection{Three-Ring Compounds}

Due to high clearing temperatures in both threering compounds (Table 1), the relaxation spectra could be collected only in a temperature region far below the clearing points. Both substances exhibited a strong conductivity at low frequencies, as shown in Figure 5. Several re-crystallization procedures were applied which did not suppressed the ionic conductivity considerably, especially in the case of compound $\mathbf{3}$. 

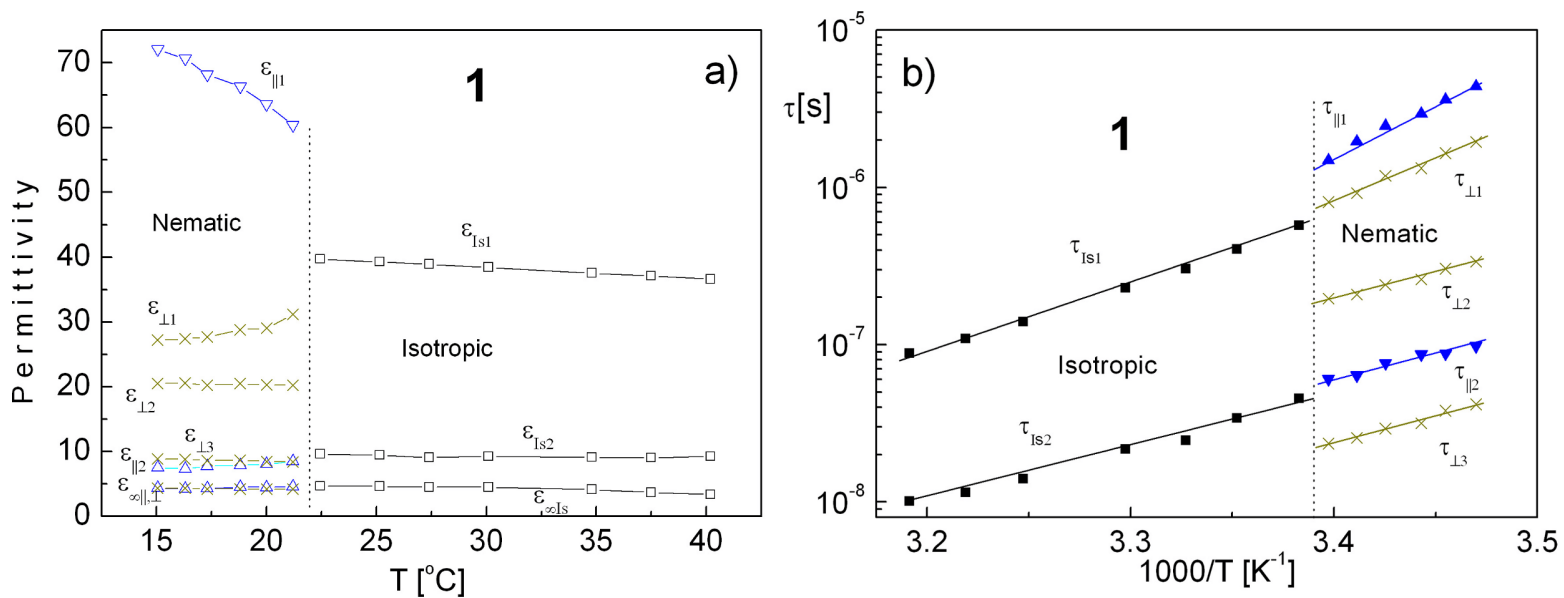

Fig. 4. Results of detailed analysis of the dielectric relaxation spectra presented in Figures 2 and 3 for the substance 1: a) dielectric parameters, b) relaxation times.
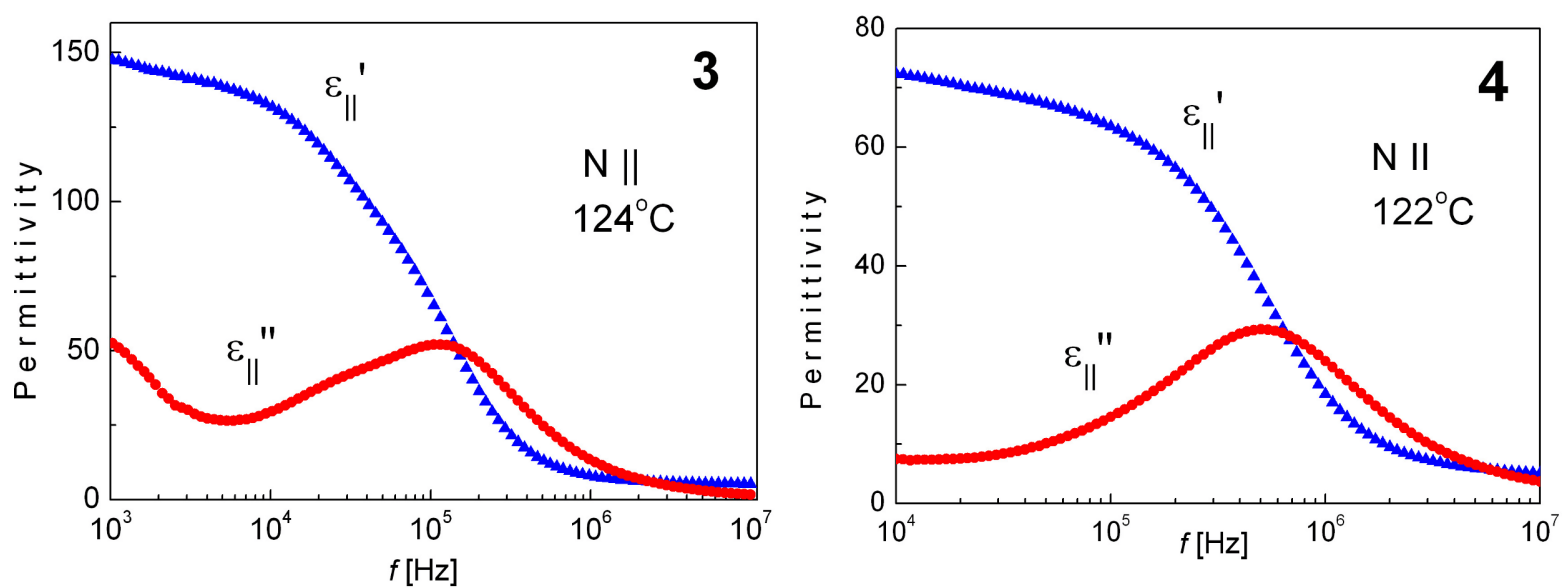

Fig. 5. Dispersion and absorption spectra for three-ring compounds in the nematic phase (parallel orientation).

The common feature of these spectra is the fact that the dispersion curve does not exhibit a plateau at low frequencies so that the static value could not be determined directly. Moreover, the $\varepsilon^{\prime}$ values seem to be too large which especially concerns sample 3. Similar effects were observed in other strongly polar LC substances as well [14]. The absorption spectra of sample 4 consist of one Debye-type relaxation process whereas for sample $\mathbf{3}$ an additional low frequency process is present which can be attributed to the doublelayer relaxation caused by free charges. In that situation we decided to establish the static permittivity values of all four substances in an indirect way by measuring their $10 \%$ wt. solutions in the Base 903 mixture (Table 1) which exhibits the small negative dielectric anisotropy $\Delta \varepsilon=\varepsilon_{\|}-\varepsilon_{\perp}<0$, see Figure 6a, bottom. In Figure $6 \mathrm{a}$ the results of the measurements for all mixtures are presented. Their clearing points are given in Table 2. Assuming that both components of the mixtures contribute additively to the permittivity, the values $\varepsilon_{I}, \varepsilon_{\|}$, and $\varepsilon_{\perp}$ characterizing the pure substances were calculated, see Figure $6 \mathrm{~b}$.

Relatively low concentrations of strongly polar molecules in the low polar solvent allow one to assume that the permittivity values displayed in Figure 6b correspond to the non-associated state of substances. In real substances a strong association of molecules can be expected which, besides of the conductivity effects, should change the permittivity values considerably. This is seen well in Figure 7 where compar- 
Table 2. Dipole moment components and the angles between different molecular axes as explained in the text and Figure 10.

\begin{tabular}{lccccccccrr}
\hline No & $\mu[\mathrm{D}]$ & $\mu_{x}[\mathrm{D}]$ & $\mu_{y}[\mathrm{D}]$ & $\mu_{z}[\mathrm{D}]$ & $\beta_{\text {chem }}$ & $\Delta \beta$ & $\beta_{\text {cal }}$ & $\beta_{\text {Obs }}$ & $\mu_{\mathrm{l}}[\mathrm{D}]$ & $\mu_{\mathrm{t}}[\mathrm{D}]$ \\
\hline $\mathbf{1}$ & 8.880 & -8.258 & -3.142 & -0.882 & 21.56 & 4.13 & 25.69 & 26.34 & 8.00 & 3.85 \\
$\mathbf{2}$ & 8.959 & -8.340 & -3.155 & -0.866 & 21.42 & 4.94 & 26.36 & 27.60 & 8.03 & 3.98 \\
$\mathbf{3}$ & 11.826 & -10.704 & -4.579 & 2.074 & 25.16 & 3.78 & 28.94 & - & 10.35 & 5.72 \\
$\mathbf{4}$ & 11.893 & -10.739 & -4.718 & 1.968 & 25.45 & 2.49 & 27.95 & - & 10.51 & 5.57 \\
\hline
\end{tabular}
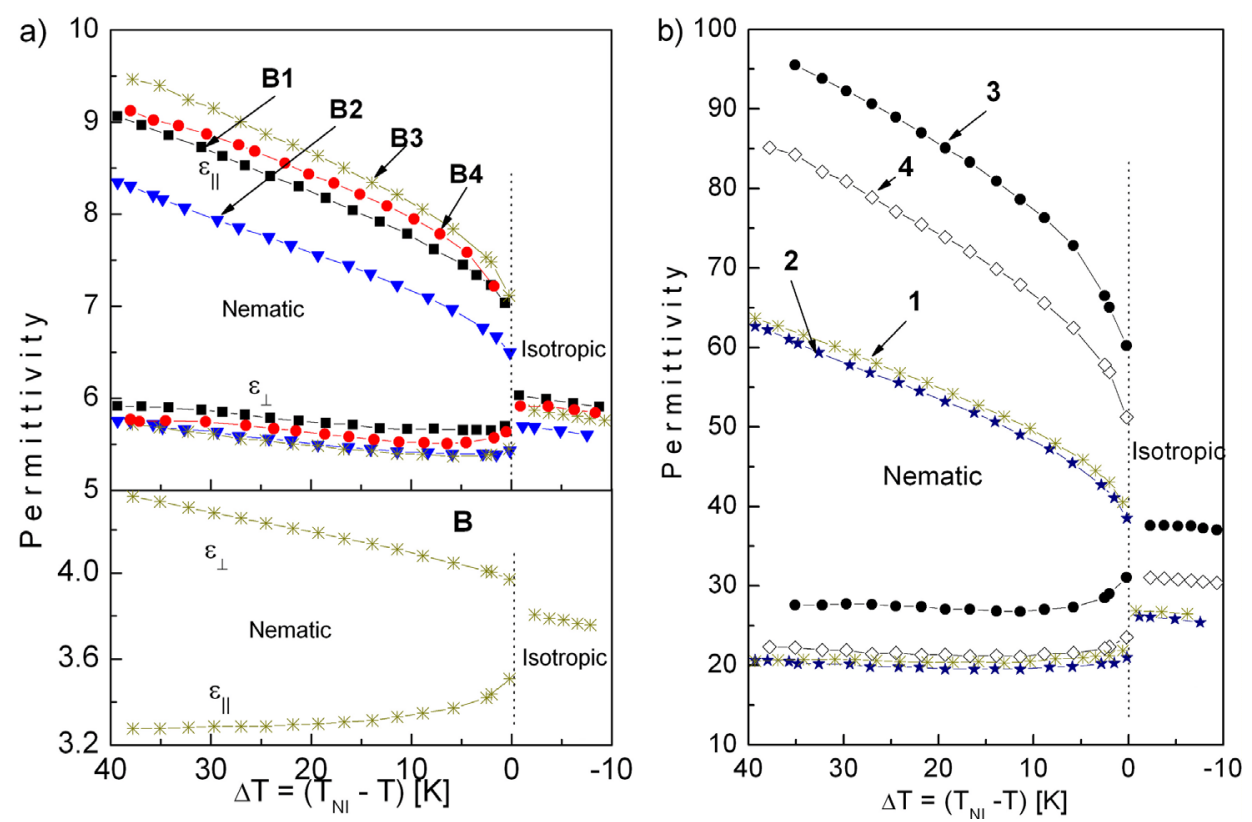

Fig. 6. (a) Tensor permittivity components measured for Base $\mathbf{9 0 3}$ and its four $10 \%$ wt. mixtures, B1 to B4, with particular substances (corresponding to $0.09577,0.08532,0.07560$, and 0.06234 of mole fraction for compounds $\mathbf{1}$ to 4 , respectively). (b) Tensor permittivity components calculated from the measurements in mixtures for the four substances.

isons of the experimental and calculated permittivity values are presented. (In the case of compound $\mathbf{4}$ the static permittivity values were obtained from the analysis of the relaxation spectra by fitting the semicircle to the points measured above c. $100 \mathrm{kHz}$ as is shown in Figure 8a). Only for compound 2 the agreement of both sets of data is fairly good. Relations between the measured and the calculated permittivities for compounds 1 and $\mathbf{4}$ are opposite which indicates the presence of parallel (1) and antiparallel (4) correlations in pure compounds. More information about those effects can be achieved if the measurements are made for several concentrations of mixtures.

\section{Discussion}

\subsection{Static Permittivities}

Several dipolar groups constitute the molecules under study. Figure 10 shows the molecule 1 simu- lated with the aid of HyperChem ${ }^{\mathrm{TM}}$ Release 7.51 and CS Chem3D Pro with CS MOPAC Pro ${ }^{\text {TM }}$ software, based on the semi-empirical AM1 method [15]. These enabled us to calculate the dipole moment components (gathered in Table 2), to determine the principal inertia moments axes (marked as 1, 2, 3 in Fig. 9) and to estimate the angle $\beta_{\text {cal }}$ between the net dipole moment and the long molecular axis (the 1-axis), as well as the angle $\beta_{\text {chem }}$ between the net dipole moment and the $x$-axis defined as the para-axis of the phenyl ring with the butyloxy chain. As can be seen in Figure 9, the para-axis differs from the 1-axis by the angle $\Delta \beta$.

The angle $\beta$ was also estimated on the basis of the experimental data. From the TDS spectra of compounds 1 and 2 presented in Figure 2 the parameters $\varepsilon_{\mathrm{s}}$ and $\varepsilon_{\infty}$ for two relaxation processes were calculated using formula (1). By applying the Onsager equa- 

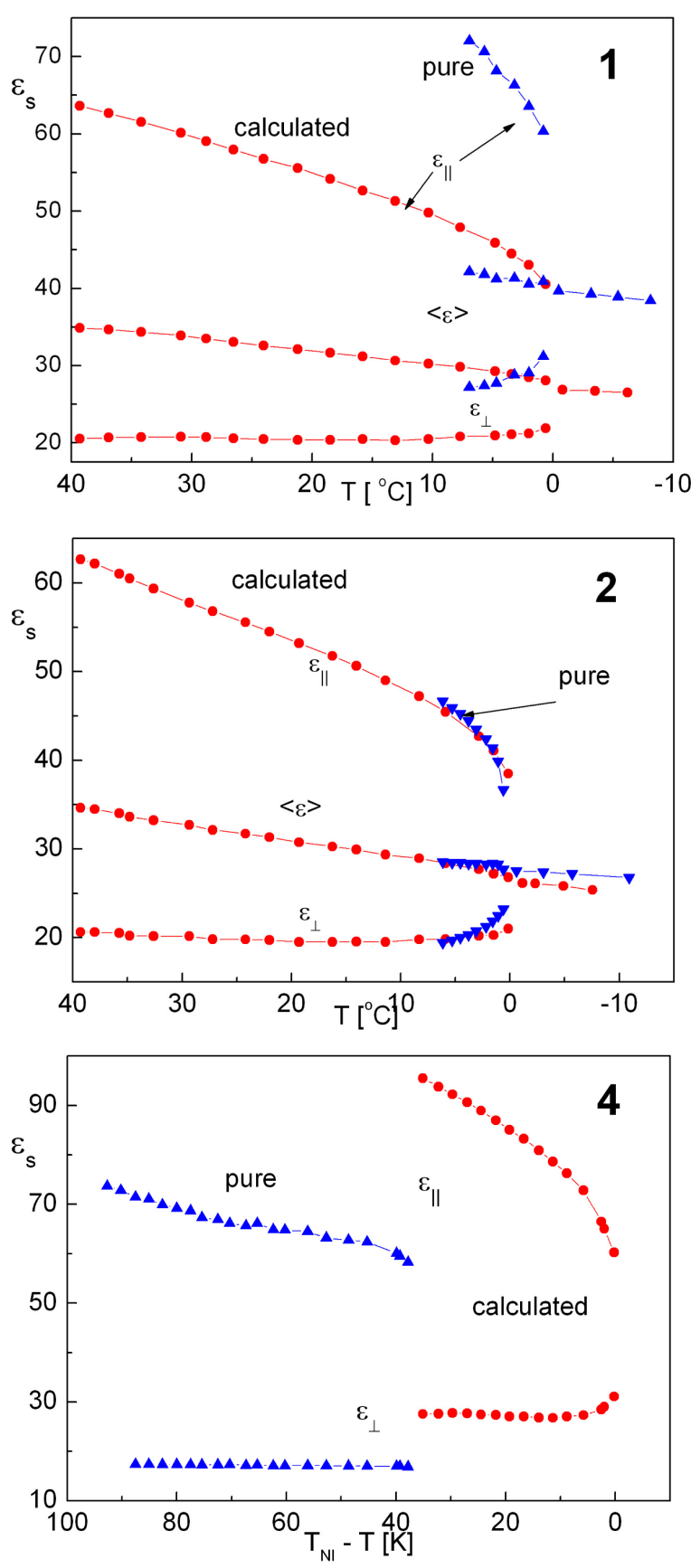

Fig. 7. Comparison of the tensor permittivity values for three substances obtained experimentally with those calculated from their 10\% wt. mixtures in Base 903.

tion [16],

$$
\frac{\left(\varepsilon_{\mathrm{s}}-\varepsilon \infty\right)\left(2 \varepsilon_{\mathrm{s}}+\varepsilon \infty\right)}{\varepsilon_{\mathrm{s}}\left(\varepsilon_{\infty}+2\right)^{2}}=\frac{N_{0}}{3 \varepsilon_{0}} \frac{\mu^{2}}{3 k T},
$$

and taking into account the fitting parameters $\varepsilon_{\mathrm{s}, \text { If. }}$. and $\varepsilon_{\infty l f}$, governed by the longitudinal component $\mu_{1}$, and $\varepsilon_{s, \mathrm{hf}}$ and $\varepsilon_{\infty, \mathrm{hf}}$, governed by the transverse component $\mu_{\mathrm{t}}$ separately, we were able to calculate the angle $\beta_{\text {Ons }}=\tan ^{-1}\left(\mu_{t} / \mu_{1}\right)$. The obtained values $\beta_{\text {Ons }}$ are in good agreement with $\beta_{\text {cal }}$ (Table 2).

As it is seen in Figure 1 and Table 3 the permittivity values differ considerably between substances $\mathbf{1}$ and $\mathbf{2}$ in spite of close dipole moments values (Table 2). The compound $\mathbf{2}$ may be treated as internal diluted medium because of its longer alkoxy chain. In the solution this effect is compensated by the low polar solvent. Therefore, the sequences of permittivities in the isotropic and $\mathrm{N}$ phases obtained from the measurements in solutions (Figure 6b) seem to be in accordance with the dipole moment values of particular substances.

Those behaviours of the permittivities may be discussed on the basis of the Onsager equation (2) (isotropic phase) or the Maier and Meier equations (3) - (5) [17] (nematic phase),

$$
\begin{aligned}
&\left(\varepsilon_{\|}-1\right)= \varepsilon_{0}{ }^{-1} N F h\left\{\bar{\alpha}+\frac{2}{3} \Delta \alpha S\right. \\
&\left.+F \frac{\mu^{2} \mathrm{eff}}{3 k T}\left[1-\left(1-3 \cos ^{2} \beta\right) S\right]\right\}, \\
&\left(\varepsilon_{\perp}-1\right)= \varepsilon_{0}^{-1} N F h\left\{\bar{\alpha}-\frac{1}{3} \Delta \alpha S\right. \\
&\left.+F \frac{\mu^{2} \mathrm{eff}}{3 k T}\left[1+\frac{1}{2}\left(1-3 \cos ^{2} \beta\right) S\right]\right\}, \\
& \Delta \varepsilon=\left(\varepsilon_{\|}-\varepsilon_{\perp}\right)= \\
& \varepsilon_{0}^{-1} N \cdot F \cdot h \cdot\left[\Delta \alpha-F \frac{\mu^{2} \mathrm{eff}}{2 k T}\left(1-3 \cos ^{2} \beta\right)\right] S,
\end{aligned}
$$

where $N=N_{\mathrm{A}} \rho / M$ with $N_{\mathrm{A}}$ the Avogadro's number, $\rho$ the density, $M$ the molar mass, $\varepsilon_{0}$ is the permittivity of free space, $\Delta \alpha=\alpha_{1}-\alpha_{\mathrm{t}}$ and $\bar{\alpha}=\left(\alpha_{1}+2 \alpha_{\mathrm{t}}\right) / 3$, $S$ is the nematic order parameter. The local field parameters $F$ and $h$ are expressed by the mean polarizability $\bar{\alpha}$ and mean permittivity $\bar{\varepsilon}=\left(\varepsilon_{\|}+2 \varepsilon_{\perp}\right) / 3$. $\mu^{2}$ eff $=g \mu^{2}$ is the effective dipole moment, and $g$ is the dipole-dipole correlation factor. The differences in the observed $\varepsilon$-values (Fig. 1) cannot be attributed to the differences in the density values that change between the members of the homologous series rather slightly: the ratio $\rho_{4} / \rho_{8} \sim 1.04$ [18], whereas the ratio $\Delta \varepsilon_{4} / \Delta \varepsilon_{8} \sim 1.6$ (see in the inset of Fig. 1) and $\varepsilon_{\mathrm{I} 4} / \varepsilon_{\mathrm{I} 8} \sim$ 1.4 in the isotropic phase. The change in the length of the butyl and octyl tails yields reduction in the $\varepsilon_{I}$ and $\Delta \varepsilon$ values by factor $\sim 1.2$ [18]. A possible difference in 

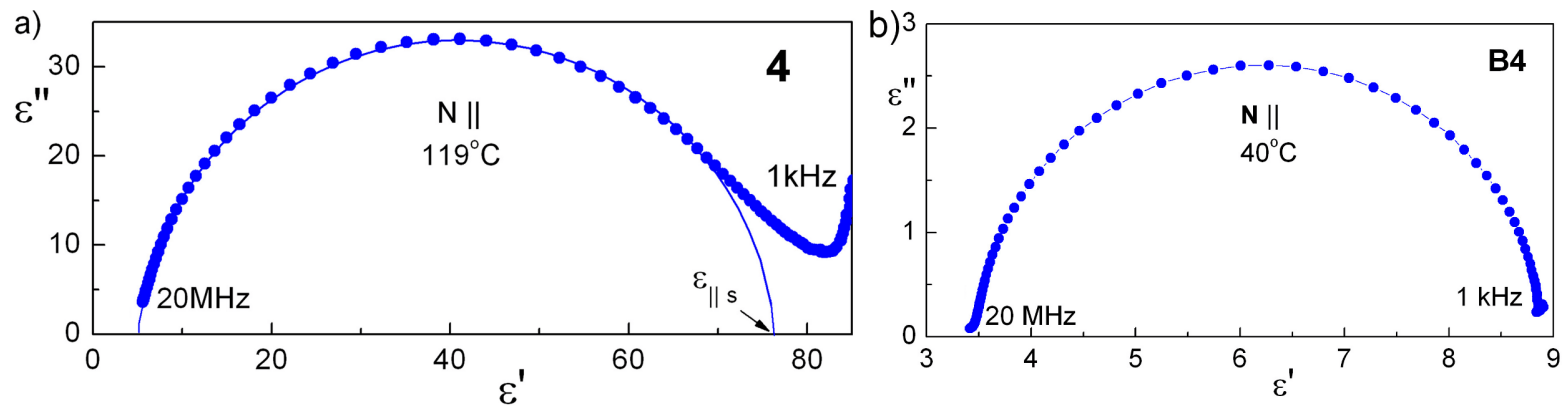

Fig. 8. Cole-Cole plots for the spectra of compound $\mathbf{4}$ in the nematic phase measured for: (a) the pure substance, (b) the $10 \%$ wt. mixture of compound $\mathbf{4}$ in the Base 903. The static $\varepsilon_{\| \text {s }}$ values presented in Figure 7 were taken from fitting the Debye semi-circle to the spectra as indicated in (a).

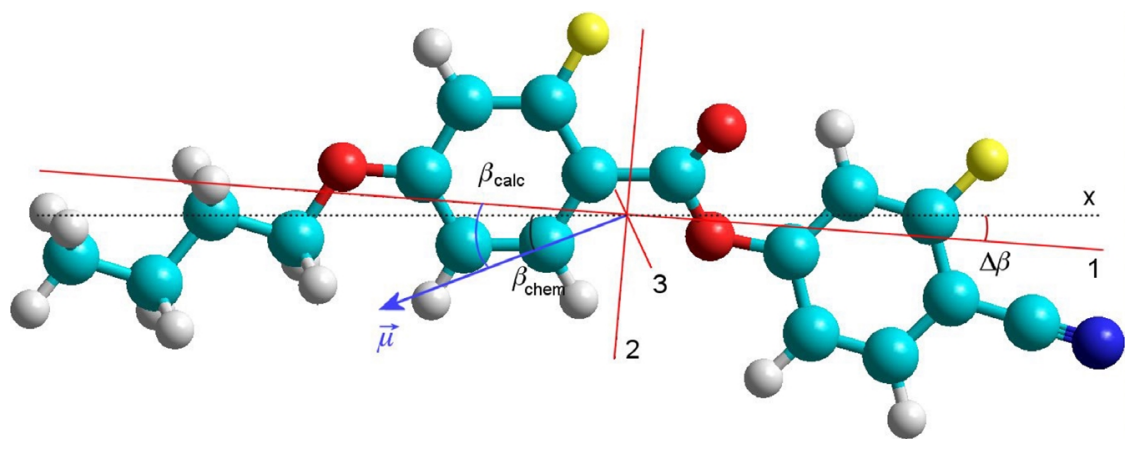

Fig. 9. Molecular structure of the substance 1 obtained with the aid of the HyperChem ${ }^{\mathrm{TM}}$ software. The reference axes and the angles listed in Table 2 are shown.

the nematic order parameter values cannot be responsible for that because the effect is pronounced in the isotropic phase as well. Taking the above into consideration, as well as the relations between the data obtained from the mixtures (Fig. 7), one can suppose that the observed effects must be attributed to the dipoledipole correlations which seem to be parallel $(g>1)$ for substance 1 and antiparallel $(g<1)$ for substance $\mathbf{4}$, with no correlations, perhaps, for substance 2 . The models describing different molecular correlations in LC phases can be found in [19-23]. In many works it was established that antiparallel pairing (dimerization) strongly increases with alkyl (hydrocarbon part) length of molecule, see example in $[24,25]$. In the case of compounds having smectic A phase, transformation of smectic $A_{1}$ subphase to smectic $A_{d}$ subphase is observed with growing alkyl chain [26]. In the near future the dielectric measurements for several concentrations of substances $\mathbf{1}$ and $\mathbf{4}$ in a non-polar nematogen will be performed in order to analyze these intermolecular interactions.

\subsection{Dielectric Relaxation}

In the isotropic phase of two-ring compounds two relaxation modes were observed by the impedance

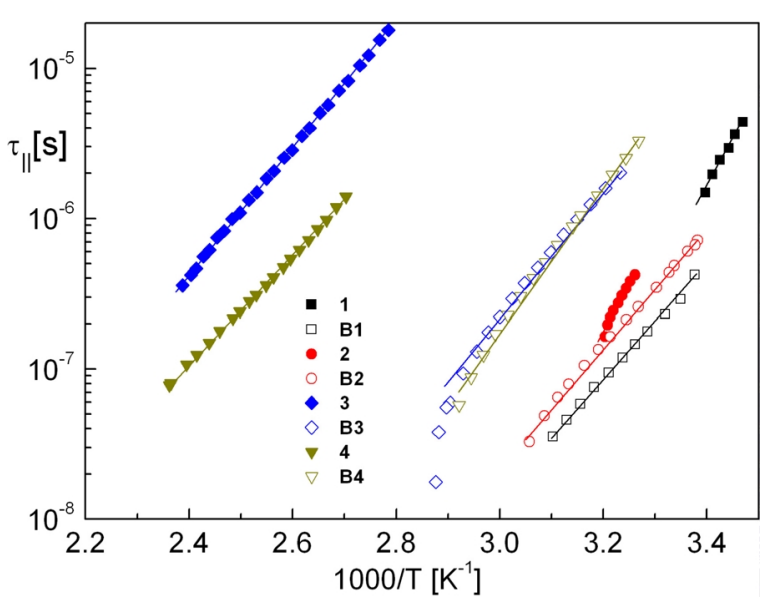

Fig. 10. Longitudinal relaxation times vs. inverted temperature determined for pure substances (full symbols) and their mixtures with the Base 903 (open symbols).

(low temperatures) and TDS (high temperatures) methods, see Figure 2. These modes can be attributed to the molecular rotations around the principal inertia axes. Superposition of two Debye-type formulae (1) fits the spectra well (especially for substance 1). It is rather unusual that these spectra are shifted to the 
Table 3. Parameters obtained from the analysis of the results of dielectric measurements for all substances. $\varepsilon_{\|}$, $\varepsilon_{\perp}$ taken at $T \sim T_{m}$ or at $\Delta T=30 \mathrm{~K}$ for the data calculated as explained in the text (marked by $*$ ). $\Delta \varepsilon$ (h.f.) taken at $f \sim 10 \times f_{\text {co. }}$ The data marked by ${ }^{M}$ correspond to the mixtures.

\begin{tabular}{|c|c|c|c|c|c|c|c|c|c|c|c|c|}
\hline \multirow[t]{2}{*}{$\overline{\mathrm{No}}$} & \multirow[t]{2}{*}{$\overline{\varepsilon_{I}}$} & \multirow[t]{2}{*}{$\varepsilon_{\|}$} & \multirow[t]{2}{*}{$\overline{\varepsilon_{\perp}}$} & \multicolumn{2}{|c|}{$\Delta \varepsilon$} & \multirow{2}{*}{$\begin{array}{c}f_{\mathrm{co}} \\
{[\mathrm{MHz}]}\end{array}$} & \multicolumn{6}{|c|}{$\Delta H[\mathrm{~kJ} / \mathrm{mol}]$} \\
\hline & & & & 1.f. & h.f. & & $\begin{array}{c}I_{1} \\
N_{\perp 3}\end{array}$ & $I_{2}$ & $N_{\| 1}$ & $N_{\| 2}$ & $N_{\perp 1}$ & $N_{\perp 2}$ \\
\hline \multirow[t]{2}{*}{1} & 39.5 & 72.1 & 27.1 & 45.0 & -7.9 & 0.06 & 81.2 & 65.3 & 120.8 & 57.2 & 101.3 & 63.9 \\
\hline & $26.7 *$ & $61.0 *$ & $20.4^{*}$ & $\mathrm{xx}^{\mathrm{M}}$ & & & 67.3 & & & & & \\
\hline \multirow[t]{2}{*}{2} & 27.5 & 46.7 & 19.6 & 27.1 & - & 0.58 & & & $72.0^{\mathrm{M}}$ & & & \\
\hline & $26.0 *$ & $58.9^{*}$ & $20.2 *$ & 5.0 & & & 70.5 & 27.4 & 118.4 & & & \\
\hline \multirow[t]{2}{*}{3} & $37.7 *$ & $93.4 *$ & $27.8 *$ & & & 0.37 & & & $75.7^{\mathrm{M}}$ & & & \\
\hline & & & & $46.4^{*}$ & - & & & & 81.2 & & & \\
\hline \multirow[t]{2}{*}{4} & $31.1 *$ & $81.9 *$ & $22.2 *$ & 6.5 & & 1.32 & & & $83.5^{\mathrm{M}}$ & & & \\
\hline & & & & & & & & & 69.1 & & & \\
\hline \multirow[t]{2}{*}{ Base 903} & 3.82 & 3.28 & 4.40 & $41.8^{*}$ & - & & & & $90.9^{\mathrm{M}}$ & & & \\
\hline & & & & 5.1 & & & & & & & & \\
\hline
\end{tabular}

kilo and megahertz frequencies; for many two-ring cyano-compounds these modes were observed at hundred $\mathrm{MHz}$ or even $\mathrm{GHz}$ frequencies [27, 28]. Besides, shifting of the process into low frequencies is stronger for the shorter molecule $\mathbf{1}$. The relaxation times presented in Figure $4 \mathrm{~b}$ yield the activation enthalpies, $\Delta H=R\left(\partial \ln \tau / \partial T^{-1}\right)$ (Table 3 ) which are distinctly larger than those obtained for typical nematogens (usually $30-40 \mathrm{~kJ} / \mathrm{mole}[4,25-30]$ ). Certainly, that is caused by the strong intermolecular interactions discussed above.

For the parallel orientation of the $\mathrm{N}$ phase in the pure substances as well as in the mixtures are described very well by the monodomain Debye formula, i. e. in the Cole-Cole plots the semicircles fit the points excellently, see Figures 3 and 8.

The relaxation spectra measured for the nematic phase of two-ring substances, Figure 3, show features typical for this class of compounds [4, 14, 27, 28]. For parallel orientation the low frequency mode corresponding to the molecular rotations around the short axis dominates the spectra of both substances, although the high frequency mode connected with the molecular rotations around the long axis is also present due to imperfect alignment of the samples. The ratio of strengths of both processes equals to $\sim 1.61$ and is markedly larger than 1.43 being the ratio of the respective dipole moment components squared (compare Maier-Meier equations (3), (4) and Table 2) which indicates that the dipole correlation factor $g_{\|}>1$. The calculated activation enthalpy values are listed in Table 3. The so-called retardation factor [31] $q=$ $\tau_{\|} / \tau_{I 1}=2.1$ and 2.0 for substances $\mathbf{1}$ and $\mathbf{2}$, respectively, and is typical for many nematogens [27, 2830, 32].
The longitudinal relaxation times determined for all substances in the $\mathrm{N}$ phase of pure state and in mixtures are presented in Figure 10 in form of the Arrhenius plot. The values of the relaxation times are similar, whereas the activation enthalpies $\Delta H_{\|}$are different (see Table 3). For two-ring compounds the barriers hindering the flip-flop molecular motions in mixtures are considerably lower than in the pure substances; this, in part, is connected with a narrow range of the $\mathrm{N}$ phase but certainly arises from the strong intermolecular interactions in pure substances. In the case of three-ring compounds both quantities are rather similar.

For perpendicular orientation the spectra measured for the compound $\mathbf{1}$ could only be properly analysed, see Figure 3. Superposition of three Debye-type semicircles fits the spectra well. They can be compared with the spectra obtained by Jadżyn et al. [32] for similar substance, 4-cyanophenyl 4- $n$-heptylbenzoate. However, in that case the modes were shifted to much higher frequencies. These authors discussed carefully the origins of three observed relaxation bands. It seems that their conclusions can be applied to the present case as well. The high frequency mode 3 was ascribed to the molecular rotations around the long axis. The mid frequency mode 2 can be associated with stochastic precessional movements and/or fluctuations of the long axis with respect to the director. The relaxation process corresponding to mode 1 is not predicted by the theoretical models. Jadzyn et al. have argued that it may results from strong dipole-dipole interactions present in the cyano-compounds. It should be mentioned that the activation enthalpy values obtained for the modes 2 and 3 of compound $\mathbf{1}$ are remarkably large (Table 3 ) whereas in the case of 4-cyanophenyl 4$n$-heptylbenzoate were negligible [32]. This indicates 

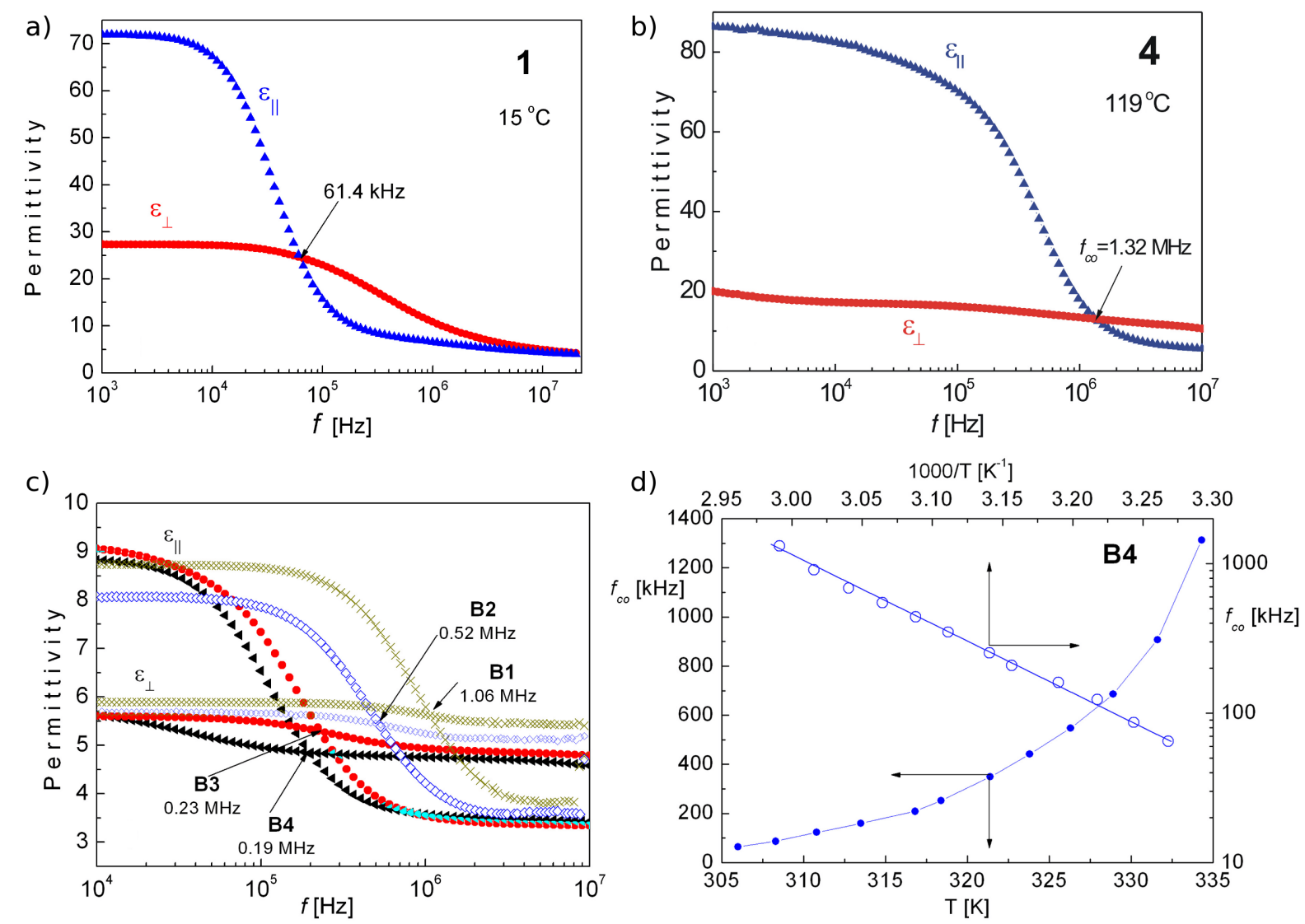

Fig. 11. Dispersion spectra recorded for two alignments of the nematic phase in pure compounds $\mathbf{1}$ (a) and $\mathbf{4}$ (b), and compounds $1-4$ in the Base 903 mixtures (c) at $\Delta T=30 \mathrm{~K}$. The crossover frequencies are indicated by arrows. (d) Temperature dependence of the cross-over frequency in one mixture in two scales; linear dependence $f_{\text {co }}$ vs. $1 / T$ indicates its exponential character.

that in the substance $\mathbf{1}$ strong intermolecular interactions hinder the molecular motions around the long axes as well.

\subsection{Cross-Over Effect}

Figure 11 shows the dispersion spectra plotted for two orientations of the nematic phase in two pure substances $(a, b)$ and in four mixtures (c). At a certain frequency, called the crossover frequency $f_{\text {co }}$, the dielectric anisotropy changes sign from positive at low frequencies to negative above $f_{\text {co }}$. The values of both anisotropies are listed in Table 3. This effect enables designing the dual addressing displays. The here investigated alkoxy compounds have higher $f_{\text {co }}$ value than recently studied analogous alkyl derivatives [8,33]. Figure 11d shows that the crossover frequency exponentially depends on the temperature with the activa- tion enthalpy slightly smaller than the value characterizing the flip-flop molecular motion in the $\mathrm{N}$ phase.

\section{Conclusions}

The four studied nematogenic compounds possess large longitudinal and transverse dipole components which make them interesting for the formulation of mixtures useful for some application. However, very large dielectric anisotropy in the nematic phase causes a dissociation of polar bonds and a distinct ionic conductivity appears which influences the dielectric properties of pure substances considerably. It was shown that the dielectric measurements of such substances diluted in low-polar medium can bring valuable data on the tensor permittivity components in the nematic phase. Differences in the dielectric properties of substances in the pure and diluted states were interpreted 
in terms of strong dipole-dipole interactions, especially in the case of two-ring compounds. Also the rotational dynamic properties of molecules provide evidence of such interactions in the isotropic and nematic phases of these compounds. More information about those inter- actions can bring further investigations performed for several concentrations of mixtures.

\section{Acknowledgement}

The work was carried under Project PBS765.
[1] W. H. de Jeu, Physical Properties of Liquid Crystalline Materials, Gordon and Breach, New York 1980.

[2] H. Kresse, Adv. Liq. Cryst. 6, 109 (1983).

[3] S. Urban, Static dielectric properties of nematics, in: Physical Properties of Liquid Crystals: Nematics (Eds. D. Dunmur, A. Fukuda, and G. Luckhurst), EMIS Datareviews Series 2001, Chapter 6.1.

[4] S. Urban, Dielectric relaxation processes in calamitic liquid crystals. Influence of temperature and pressure, in: Dielectric Properties of Liquid Crystals (Eds. Z. Galewski and L. Sobczyk), Transworld Research Network, Kerala, India 2007, Chapter 10.

[5] E. Lueder, Liquid Crystal Displays: Addressing Schemes and Electrooptical Effects, Wiley SID Series in Display Technology, John Wiley \& Sons Ltd., The Atrium, Southern Gate, Chichester, England 2004.

[6] H. Stettin, H. Kresse, and W. Schafer, Cryst. Res. Technol. 24, 111 (1989).

[7] T. Geelhaar, Liq. Cryst. 24, 91 (1998).

[8] D. Demus, Y. Goto, S. Sawada, E. Nakagawa, H. Saito, and R. Tarao, Mol. Cryst. Liq. Cryst. 260, 1 (1995).

[9] G. Heppke, J. Kayed, and U. Mueller, Mol. Cryst. Liq. Cryst. 98, 309 (1982).

[10] D. Ziobro, R. Dạbrowski, P. Kula, J. Dziaduszek, M. Filipowicz, J. Parka, J. Czub, S. Urban, and S.-T. Wu, Opto-Electronics 17, 16 (2009).

[11] B. Jankiewicz, W. Drzewiński, and R. Dąbrowski, Conference Proceedings, XVI Conference on Liquid Crystals, Stare Jabłonki 2005, Poland, p. 21 - 28 (2007).

[12] B. Gestblom, in: Relaxation Phenomena, (Eds. W. Haase and S. Wróbel), Springer-Verlag, Berlin 2003, Chapter 1.2.

[13] K.S. Cole and R.H. Cole, J. Chem. Phys. 9, 341 (1949).

[14] K. L. Sandhya, D. S. Shankar Rao, S. Krishna Prasad, U.S. Hiremath, and C. V. Yelamaggad, Thermochim. Acta 452, 65 (2007).

[15] HyperChemTM, Release 7.51, 2002 Hypercube, Inc.
[16] A. Chelkowski, Dielectric Physics, Elsevier, Amsterdam 1980.

[17] W. Maier and G. Meier, Z. Naturforsch. 16a, 262, 470 (1961).

[18] P. Sarkar, P. Mandal, S. Paul, R. Paul, R. Dąbrowski, and K. Czuprynski, Liq. Cryst. 30, 507 (2003).

[19] A. J. Leadbetter, R. M. Richardson, and C. N. Colling, J.de Physique 36, C1-37 (1975).

[20] L. Longa and W. H. de Jeu, Phys. Rev. A 26, 1632 (1982).

[21] R. Berardi, S. Orlandi, and C. Zannoni, Chem. Phys. Lett. 261, 357 (1996).

[22] N. V. Madhusudana, Braz. J. Phys. 28, n.4, Săo Paulo Dec. 1998.

[23] A. G. Vanakaras and D. J. Photinos, Mol. Cryst. Liq. Cryst. 395, 213 (2003).

[24] P. Sarkar, P. Mandal, S. Paul, R. Paul, R. Dąbrowski, and K. Czupryński, Mol. Cryst. Liq. Cryst. 330, 159 (1999).

[25] R. Dąbrowski, J. Jadżyn, J. Przedmojski, and J. Baran, Cryst. Res. Technol. 22, 861 (1987).

[26] R. Dạbrowski, K. Pyc, J. Przedmojski, and B. Pura, Mol. Cryst. Liq. Cryst. 129, 169 (1985).

[27] J. Jadżyn, G. Czechowski, R. Douali, and Ch. Legrand, Liq. Cyst. 26, 1591 (1999).

[28] J. Jadżyn, L. Hellemans, G. Czechowski, C. Legrand, and R. Douali, Liq. Cyst. 27, 613 (2000).

[29] S. Urban, B. Gestblom, and A. Würflinger, Mol. Cryst. Liq. Cryst. 331, 113 (1999).

[30] J. Czub, U. Gubernat, B. Gestblom, R. Dạbrowski, and S. Urban, Z. Naturforsch. 59A, 316 (2004).

[31] M. Martin, G. Maier, and A. Saupe, Discussion Faraday Soc. 5, 119 (1971).

[32] J. Jadżyn, Ch. Legrand, G. Czechowski, and D. Bauman, Liq. Cyst. 24, 689 (1998).

[33] D. Ziobro, J. Dziaduszek, M. Filipowicz, R. Dạbrowski, J. Czub, and S. Urban, Mol. Cryst. Liq. Cryst. 52, 258 (2009). 